\title{
GPPS-CH-2020-0039
}

\section{VALIDATION OF A HIGH-ORDER NUMERICAL DISCRETIZATION SCHEME FOR THE SOLUTION OF THE 2-D EULER EQUATIONS}

\author{
Dimitrios P. Angelopoulos \\ Technical University of Crete, \\ School of Production \\ Engineering and Management \\ daggelopoulos@isc.tuc.gr \\ Chania, Crete, Greece
}

\author{
Georgios N. Lygidakis \\ Technical University of Crete, \\ School of Production \\ Engineering and Management \\ glygidakis@isc.tuc.gr \\ Chania, Crete, Greece
}

\author{
loannis K. Nikolos \\ Technical University of Crete, \\ School of Production \\ Engineering and Management \\ jnikolo@dpem.tuc.gr \\ Chania, Crete, Greece
}

\begin{abstract}
A recently-proposed high-order numerical discretization scheme enhances an in-house solver for the 2D/2Daxisymmetric Euler and Navier-Stokes equations, discretized with a Node-Centered Finite Volume method on triangular or hybrid unstructured girds. The adopted high-order scheme is based on the incorporation of additional high order terms to the reconstructed nodal values, used for the computation of the inviscid fluxes. The required higher-order derivatives are computed with the corresponding lower-order ones on the existing Degrees-of-Freedom via a successive differentiation technique. As a result, the connectivity requirements are restricted to the first neighbouring points. The aforementioned technique was incorporated to the $U-M U S C L$ variable extrapolation numerical scheme, coupled with a high-order temporal discretization that employs a Strong Stability Preserving Runge-Kutta method. To assess the effectiveness of the aforementioned numerical scheme, the 2D Euler solver is used against a benchmark test case having a smooth analytic solution. Satisfactory results are obtained for regular unstructured grids, demonstrating the proposed scheme's potential to increase the solution's accuracy for a given grid density.
\end{abstract}

\section{INTRODUCTION}

Computational Fluid Dynamics (CFD) is an everadvancing multidisciplinary scientific field, providing sufficiently accurate numerical results for various types of fluid models. It has been developed into a very powerful procedure that has been routinely applied in a wide range of industrial and non-industrial application areas ever since (Spalart and Venkatakrishnan, 2016). Notwithstanding the considerable ongoing evolution, CFD still faces several challenges that need to be addressed. Therefore, although various academic and commercial flow solvers have been developed in the past years, many issues are still subjects of continuous research (Spalart and Venkatakrishnan, 2016).
A primary concern that engages research activity in the CFD community, while also being the subject of this study, is the efficiency of the solvers in producing more accurate numerical solutions over complex configurations. It is well known that the majority of the commercial unstructured CFD codes do not provide much more than a second-order accuracy. In recent years, the focus of interest has been shifted to high-order scheme development, owing to the fact that such approaches offer greater accuracy in a given grid density (Yang and Harris, 2016).

Nevertheless, most popular methodologies, e.g. the kexact scheme (Barth, 1993), necessitate for extra information beyond the first neighbouring cells to compute high-order reconstructed values. Unlike structured solvers, where node connectivity between neighbouring grid points is implied, the calculation of the higher derivatives poses limitations for the unstructured ones, due to the lack of explicit connectivity beyond the first neighbours. On the other hand, in the Discontinuous Galerkin method (Persson, 2012) - a formulation different from the classical Finite Volume approach - this constraint is managed by introducing extra DoFs (Degrees of Freedom) in each cell to fit a high-order polynomial solution, thus introducing a great amount of complexity. Consequently, the implementation of such methodologies into existing CFD codes requires, usually, substantial modifications, especially in parallelization strategies, where the interventions on the code structure might prove to be rather laborious. Furthermore, the increased turnaround time of the numerical solution, associated with most high-order schemes, is a limiting factor for a wider spread of use as, in many practical scenarios, the computational cost is prohibiting.

The high-order scheme applied and tested in this work, relies on the incorporation of the high-order correction terms to the reconstructed nodal values, used for the computation of the inviscid fluxes, as proposed in (Yang et al., 2014; Yang and Harris, 2016). The required higher-order 
derivatives are computed with the corresponding lower-order ones on the existing DoFs, via a successive differentiation technique and, consequently, the connectivity requirements are restricted to the first neighbouring points (Yang et al., 2014; Yang and Harris, 2016). This is made feasible by exploiting the fundamental properties of the Green-Gauss theorem, overcoming the difficulty of unstructured flow solvers to retrieve information on a wider computational stencil. In this way, not only an improvement of the solution accuracy is achieved but the computational effort and memory requirements are retained at a reasonable level. This approach, thus, seems to be particularly appealing for incorporation to an existing CFD code, with only minor adjustments compared to other methodologies, especially if a domain decomposition approach is used in the code for parallel processing.

In the present study, a third-order reconstruction module is applied for the numerical solution of 2D Euler Equations. This module was integrated into an in-house compressible flow solver. The discrete form of the governing equations is solved with a Node-Centered Finite-Volume scheme, whereas for the computation of the inviscid fluxes an upwind method, applying Roe's approximate Riemann solver, is employed. High-order spatial accuracy is based on the $U$ MUSCL scheme (Burg, 2005), which closely resembles the traditional MUSCL one (van Leer, 1979). The time advancement of the aforementioned equations is achieved with an explicit scheme, using a Strong Stability Preserving $(S S P)$, five stage fourth-order Runge-Kutta method (SSP-RK $(5,4))$.

To demonstrate the effectiveness of the developed methodology, the aforementioned solver is used against a benchmark test case with a well-known analytical solution. This problem concerns the transport of an isentropic vortex in inviscid compressible flow. An extensive evaluation of the numerical solution was conducted, using a controlled environment through a successive grid refinement procedure for different types of triangular grids. Satisfactory results were obtained for regular grids, demonstrating the scheme's potential to increase the solution's accuracy for a given grid density.

The remainder of the paper is organized as follows: Next section is devoted to a brief description of the $2 \mathrm{D}$ fluid model, including the mathematical and numerical modelling of the Euler equations. Following this, the high-order method, the $U-M U S C L$ scheme and the SSP-RK method are presented. Subsequently, the numerical results of the convergence studies are reported. The paper is concluded with the discussion of the major outcomes from this work.

\section{FLOW MODELING}

\section{Governing Equations}

The motion of the fluid in two dimensions for a compressible inviscid flow is described by the Euler equations, a simplified form of the Navier-Stokes equations where viscosity and thermal conductivity are assumed equal to zero (Blazek, 2001). The differential form arranged into convective (inviscid) and source terms is expressed by the following equation (Lygidakis and Nikolos, 2015):

$$
\frac{\partial \vec{W}}{\partial t}+\frac{\partial \vec{F}^{i n v}}{\partial x}+\frac{\partial \vec{G}^{i n v}}{\partial y}=\vec{S}
$$

The conservative variables' vector $\vec{W}$, the inviscid flux vectors $\vec{F}^{i n v}, \vec{G}^{i n v}$ and the vector of the source term $\vec{S}$ are expressed in terms of the primitive variables $\rho, u, v, p$. Considering the source term as equal to zero for $2 \mathrm{D}$ problems, the conservative variables' and inviscid vectors are determined as shown in the following equations (Lygidakis and Nikolos, 2015)

$$
\vec{W}=\left[\begin{array}{c}
\rho \\
\rho u \\
\rho v \\
\rho e_{T}
\end{array}\right], \vec{F}^{i n v}=\left[\begin{array}{c}
\rho u \\
\rho u^{2}+p \\
\rho u v \\
\left(\rho e_{T}+p\right) u
\end{array}\right], \vec{G}^{i n v}=\left[\begin{array}{c}
\rho v \\
\rho v u \\
\rho v^{2}+p \\
\left(\rho e_{T}+p\right) v
\end{array}\right]
$$

where $\rho$ is the density of the fluid, $p$ is the pressure, $e_{T}$ is the specific total energy, and $\vec{V}=[u, v]^{\mathrm{T}}$ is the velocity vector. The state equation for a perfect gas is used to close the system.

\section{Spatial Discretization and Flux Computation}

The Finite Volume approach requires decomposing the computational domain $\Omega \subset \mathbb{R}^{2}$ into a set of non-overlapping control volumes and the numerical integration over each control volume. In the Node-Centered Finite Volume Scheme (NCFV) used in this work, solution values are defined at the mesh nodes (data points). An initial decomposition of the computational domain into grid elements, the so-called primal mesh, is used by the median dual partition to generate non-overlapping control volumes for the node-discretization. These control volumes are formed by connecting the centre of each cell to the midpoint of the cell edges (Fig. 1) and cover the entire computational domain, composing a mesh that is dual to the primal mesh (Kallinderis and Ahn, 2005; Lygidakis and Nikolos, 2015).

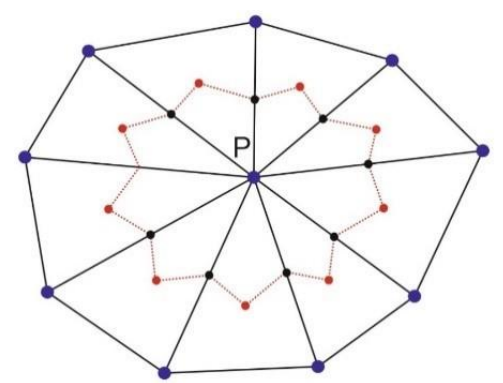

Figure 1 Control Volume of a Node $P$

Taking into account the previously described spatial discretization, the integration of Euler equations over the control volume $C E_{P}$, along with the employment of the Green-Gauss divergence theorem, leads to 


$$
\iint_{C E_{P}} \frac{\partial \vec{W}}{\partial t} d x d y+\iint_{\partial C E_{P}} \overrightarrow{\hat{H}}^{i n v} d l=0
$$

where $\partial C E_{P}$ denotes the boundaries of the control volume of node $P$, defined by the facets constructed around the edges connecting node $P$ with each neighbouring node $Q$. Furthermore, $\overrightarrow{\hat{H}}^{i n v}$ is the vector of the inviscid numerical fluxes and is evaluated at the midpoint of an edge connected to node $P$. This midpoint "coincides" with the interface between the adjacent control volumes of nodes $P$ and $Q$ connected with this edge.

Presuming that the conservative variables at node $P$ are equal to their mean values over the $C E_{P}$ and expressing the numerical fluxes as summations of fluxes through the faces composing the control volume of node $P$, Eq. (3) is transformed as

$$
\left(\frac{d \vec{W}}{d t}\right)_{P} E_{P}+\sum_{Q \in K_{N}(P)} \vec{\Phi}_{P Q}^{i n v}+\sum_{\left(K_{\text {out }} \in \partial C E_{P} \cap \Gamma\right)} \vec{\Phi}_{P, \text { out }}^{\text {inv }}=0 .
$$

The computation of the numerical inviscid fluxes is achieved considering a one-dimensional Riemann problem. This is performed in the direction of each normal vector that corresponds to every particular face forming the control volume of node $P$. Moreover, an upwind scheme, using Roe's approximate Riemann solver, is implemented (Roe, 1981), due to the expensive amount of calculations that the exact solution requires. Eventually, the inviscid fluxes are evaluated in the middle point of an edge $P Q$, based on the vectors of the conservative variables on the left and right side of the edge $P Q$, respectively. For conventional second-order accurate schemes, left and right states of an edge $P Q$ are reconstructed with Taylor series expansions, which take into account the corresponding values of their first neighboring mesh nodes.

A common approach incorporates the traditional MUSCL reconstruction scheme (Van Leer, 1979), using appropriate limiters, e.g. Van Albada -Van Leer (Van Albada, 1982) or Min-mod (Sweby, 1984), to control the total variation. In this context, the primitive or conservative variables $U$ of each state at the midpoint of an edge $P Q$ are approximated as following (Barth, 1992; Lygidakis and Nikolos, 2015)

$$
\begin{aligned}
& U_{P Q}^{L}=U_{P}+\frac{1}{2}(\nabla U)^{L} \vec{r}_{P Q} \\
& U_{P Q}^{R}=U_{Q}-\frac{1}{2}(\nabla U)^{R} \vec{r}_{P Q}
\end{aligned}
$$

The quantities marked by $L$ and $R$ superscripts denote the values of the variables taken at the left and right side of the interface between the nodes $P$ and $Q$, while $\vec{r}_{P Q}$ is the vector connecting these nodes and is directed from $P$ to $Q$. The extrapolation gradients $(\nabla U)^{L}$ and $(\nabla U)^{R}$ are equal to the gradients $(\nabla U)_{P}$ and $(\nabla U)_{Q}$ at nodes $P$ and $Q$ respectively, and in this study are calculated with the employment of the Green-Gauss linear representation method (Barth, 1992).

\section{HIGH-ORDER SCHEME}

\section{High-order formulation}

In this work, a recently-proposed, modular high-order spatial discretization scheme, with low-dissipation flux difference-splitting is applied and tested (Yang et al., 2014; Yang and Harris, 2016). According to this approach, no increase in DoFs within each cell is required. The core idea is the achievement of high-order accuracy by incorporating additional high-order correction terms to the discretized equations. To this end, a wider stencil of cells is successively incorporated in the process of the derivatives' computation. The data points of the cells involved in the computation of each derivative order is demonstrated in Fig. 2, where the coloured points indicate the neighbouring cells used for the successive computation of the first, second, third, and fourth derivatives, respectively.

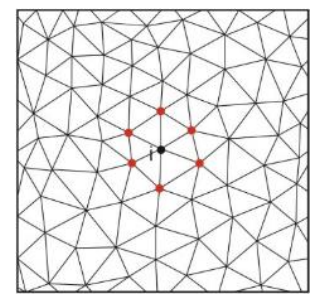

$\mathbf{1}^{\text {st }}$ Derivative

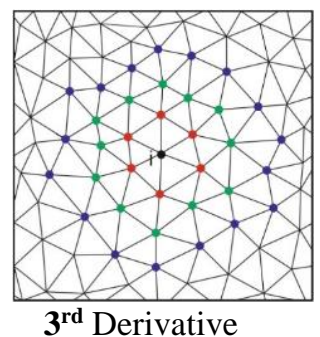

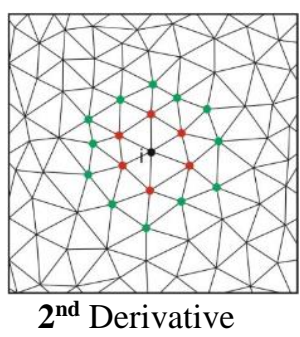

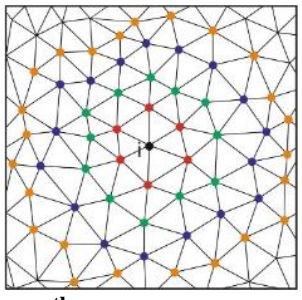

$4^{\text {th }}$ Derivative

\section{Figure 2 Stencils Used for the Computation of Derivatives}

It is worth noting that the work of (Diskin and Thomas, 2007) shows that implementing the Green-Gauss formula results in accuracy deterioration by one order for every consecutive application of the formula. Therefore, the introduced procedure may not give high-order accuracy on general unstructured grids; nonetheless, it is easier to apply onto a standing CFD code than the DG method, so that the accuracy of the base second-order scheme is improved.

\section{U-MUSCL Scheme}

The aforementioned high-order formulation was combined with the variable-extrapolation $U$-MUSCL scheme. This formulation, reported initially in (Burg, 2005), is based on information currently available to the unstructured flow solvers, namely the variable and gradient information. $U$ MUSCL closely resembles the traditional MUSCL scheme (Van Leer, 1979) and it is trivial to implement within most finite volume flow solvers. According to it, the extrapolation function in Eq. (5) is replaced by the following formulation 


$$
\begin{aligned}
& U_{P Q}^{L}(\kappa)=U_{P}+\frac{\kappa}{2}\left(U_{Q}-U_{P}\right)+\frac{1}{2}(1-\kappa) \nabla U_{P} \vec{r}_{P Q} \\
& U_{P Q}^{R}(\kappa)=U_{Q}+\frac{\kappa}{2}\left(U_{P}-U_{Q}\right)-\frac{1}{2}(1-\kappa) \nabla U_{Q} \dot{r}_{P Q}
\end{aligned}
$$

where $\kappa$ is the $U$-MUSCL parameter, $U_{P}$ and $U_{Q}$ are the left and right nodes' values of variable $U$ and $\vec{r}_{P Q}$ is the vector connecting these nodes from point $P$ to $Q$.

A one-parameter family of equations is represented in this new variable extrapolation formulation, which under certain circumstances becomes identical to the MUSCL scheme (Burg, 2005). In the case of setting $\kappa$ to 0 , the original unstructured formulation for 2nd-order variable extrapolation is obtained. In the case of setting $\kappa \tau \mathrm{o}-1$, the 2nd-order fully upwind $M U S C L$-type variable extrapolation is obtained. In the case of setting $\kappa \tau$ 七o $1 / 2$, a 3rd-order variable extrapolation to the cell face is made possible, whereas when $\kappa$ is set to $1 / 3$, a 3rd-order approximation to the derivative at the node is obtained. If $\kappa$ is set to $l$, a central difference scheme is achieved. Provided that $\kappa<1$, this formula is an upwind one, becoming stable for hyperbolic systems of equations not containing shocks, and for highquality grids. The following Table 1 summarizes what described above.

\begin{tabular}{cl}
\hline PARAMETER $(\boldsymbol{\kappa})$ & \multicolumn{1}{c}{ DESCRIPTION } \\
\hline$-\mathbf{1}$ & Second-order MUSCL-type scheme \\
\hline $\mathbf{0}$ & $\begin{array}{l}\text { Second-order unstructured } \\
\text { upwind scheme }\end{array}$ \\
\hline $\mathbf{1 / 3}$ & Third-order MUSCL-type scheme \\
\hline $\mathbf{1 / 2}$ & Third-order extrapolation to face \\
\hline $\mathbf{1}$ & Central-difference formula \\
\hline
\end{tabular}

Table 1 U-MUSCL Parameter Effect

In a similar fashion, Eq. (5) can be written in the form of Eq. (6), resulting finally in Eq. (7), where a 3rd-order scheme is incorporated (Yang and Harris, 2016). In the present work the parameters $\kappa$ and $\kappa_{3}$ are defined as $-1 / 6$ and $-4 / 3$ respectively (Yang and Harris, 2016), which correspond to a $4^{\text {th }}$ order scheme (theoretically) with no dissipation.

$U_{P Q}^{L}(\kappa)=U_{P}+\frac{\kappa}{2}\left(U_{Q}-U_{P}\right)+\frac{1}{2}(1-\kappa) \nabla U_{P} \cdot \vec{r}_{P Q}$

$+\frac{1}{2}\left[\frac{\kappa_{3}}{4}\left(\nabla U_{Q} \cdot \vec{r}_{P Q}-\nabla U_{P} \cdot \vec{r}_{P Q}\right)+\frac{1}{4}\left(1-\kappa_{3}\right) \nabla\left(\nabla U_{P} \cdot \vec{r}_{P Q}\right) \cdot \vec{r}_{P Q}\right]$

$=U_{P}+\frac{\kappa}{2}\left(U_{Q}-U_{P}\right)+\frac{1}{2}(1-\kappa) \nabla U_{P} \cdot \vec{r}_{P Q}$

$+\frac{1}{2}\left[\frac{\kappa_{3} \Delta x_{P Q}}{4}\left(\left(\frac{\partial U}{\partial x}\right)_{Q}-\left(\frac{\partial U}{\partial x}\right)_{P}\right)+\frac{1}{4}\left(1-\kappa_{3}\right) \Delta x_{P Q} \nabla\left(\left(\frac{\partial U}{\partial x}\right)_{P}\right) \cdot \vec{r}_{P Q}\right]$ $+\frac{1}{2}\left[\frac{\kappa_{3} \Delta y_{P Q}}{4}\left(\left(\frac{\partial U}{\partial y}\right)_{Q}-\left(\frac{\partial U}{\partial y}\right)_{P}\right)+\frac{1}{4}\left(1-\kappa_{3}\right) \Delta y_{P Q} \nabla\left(\left(\frac{\partial U}{\partial y}\right)_{P}\right) \cdot \vec{r}_{P Q}\right]$

According to the previous formulation, what can be noted is that that an existing code structure for a second- order scheme is capable to compute the high-order derivatives simply by calling the same routine used for the calculation of the first-order ones. Regarding the limiting procedure for the $U$-MUSCL scheme, it is under investigation.

\section{Time Integration}

Time integration is performed using a five-stage fourthorder Strong Stability Preserving Runge-Kutta method $((\operatorname{SSP}-R K(5,4))$, described as follows (Gottlieb, 2005; Ruuth, 2006)

$$
\begin{aligned}
& U_{P}^{(0)}=U_{P}^{n} \\
& U_{P}^{(i)}=\sum_{k=0}^{i-1}\left(a_{i, k} U_{P}^{(k)}\right)+\Delta t_{p} \beta_{i, k} R\left(U_{P}^{(k)}\right), a_{i, k} \geq 0, \quad i=1, \ldots, m \\
& U_{P}^{n+1}=U_{P}^{(m)}
\end{aligned}
$$

where $m$ is the number of steps and $\Delta t_{P}$ is the local time step at node $P$. Stability is assured with $C F L$ coefficient equal to 1.508 while effective $C F L_{\text {eff }}$ is equal to 0.377 . An analytic expression, along with the appropriate coefficients of the optimal $\operatorname{SSP}-R K(5,4)$, is presented as follows:

$$
\begin{aligned}
U_{P}^{(1)}= & U_{P}^{n}+0.391752226571890 \Delta t_{P} R\left(U_{P}^{n}\right) \\
U_{P}^{(2)}= & 0.444370493651235 U_{P}^{n}+0.555629506348765 U_{P}^{(1)} \\
& +0.368410593050371 \Delta t_{P} R\left(U_{P}^{(1)}\right) \\
U_{P}^{(3)}= & 0.620101851488403 U_{P}^{n}+0.379898148511597 U_{P}^{(2)} \\
& +0.251891774271694 \Delta t_{P} R\left(U_{P}^{(2)}\right) \\
U_{P}^{(4)}= & 0.178079954393132 U_{P}^{n}+0.821920045606868 U_{P}^{(3)} \\
& +0.544974750228521 \Delta_{P} R\left(U_{P}^{(3)}\right) \\
U_{P}^{n+1}= & 0.517231671970585 U_{P}^{(2)}+0.096059710526147 U_{P}^{(3)} \\
& +0.063692468666290 \Delta t_{P} R\left(U_{P}^{(3)}\right) \\
& +0.386708617503269 U_{P}^{(4)} \\
& +0.226007483236906 \Delta t_{P} R\left(U_{P}^{(4)}\right)
\end{aligned}
$$

In the numerical simulations of the convergence study that follows, $C F L$ was set to 1.5 .

\section{NUMERICAL TESTS AND RESULTS}

\section{Test Case}

An evaluation of the proposed high-order scheme was performed through a benchmark problem with a well-known analytic solution. The main goal was to test whether the proposed scheme can achieve the desired 3rd-order, for different types of unstructured grids, comprised of triangular elements. As a verification test the transport of an isotropic vortex is examined. The particular problem is characterized by smoothness with the absence of discontinuities. Therefore, no limiting functions are needed for the reconstruction.

On a computational domain $\Omega=[-10,10] \times[-10,10]$ a vortex with initial centre $\left(x_{c}, y_{c}\right)=(0,0)$ is moving from left to right in a diagonal direction. The initial solution is given by the following equations (Yang and Harris, 2016) 


$$
\begin{aligned}
& u=u_{\infty}-\beta u_{\infty} \frac{y-y_{c}}{R} \exp \left(\frac{1-r^{2}}{2}\right) \\
& v=v_{\infty}+\beta u_{\infty} \frac{x-x_{c}}{R} \exp \left(\frac{1-r^{2}}{2}\right) \\
& \rho=\rho_{\infty}\left[1-\frac{\gamma-1}{2 \gamma}\left(\beta u_{\infty}\right)^{2} \exp \left(1-r^{2}\right)\right]^{\frac{1}{1-\gamma}} \\
& p=p_{\infty}\left[1-\frac{\gamma-1}{2 \gamma}\left(\beta u_{\infty}\right)^{2} \exp \left(1-r^{2}\right)\right]^{\frac{1}{1-\gamma}} \\
& r=\sqrt{\left(x-x_{c}\right)^{2}+\left(y-y_{c}\right)^{2}} / R, \beta=1 / 2 \pi
\end{aligned}
$$

where $r$ denotes the distance from the vortex core, $R$ refers to the vortex radius and the subscript ' $\infty$ ' express the uniform mean flow. In this study, the non-dimensionalized variables are set as $u_{\infty}=1, v_{\infty}=1, \rho_{\infty}=1, p_{\infty}=1$ while periodic boundary conditions are imposed in the $x$ - and $y$-direction.

\section{Computational Grids}

The grids used in this study can be categorized as either Regular or Irregular. Regular grids are derived by a smooth mapping from grids with periodic node connectivity and periodic cell distribution, including grids derived from Cartesian ones (Delis et al., 2011; Delis and Nikolos, 2013). Four types of grids are considered in the present study: Equilateral Triangular Grids (Type I), Orthogonal Grids (Type II), Orthogonal Grids (Type III) and Distorted Grids (Type IV).

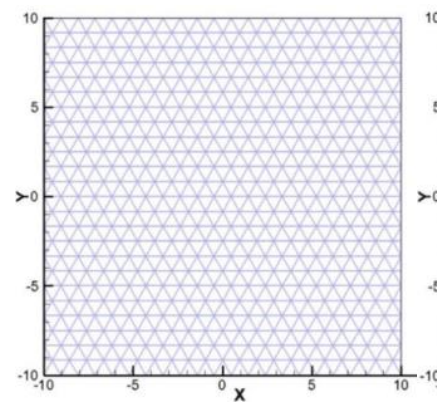

Equilateral Grid (Type I)

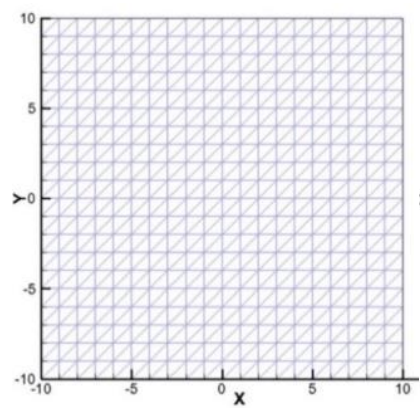

Orthogonal Grid (Type III)

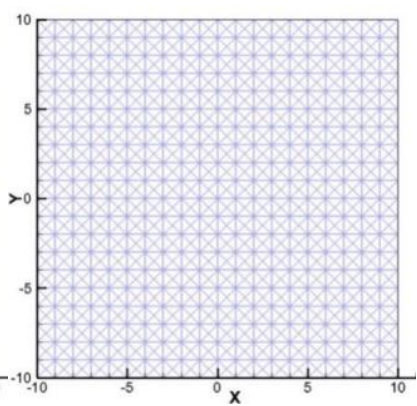

Orthogonal Grid (Type II)

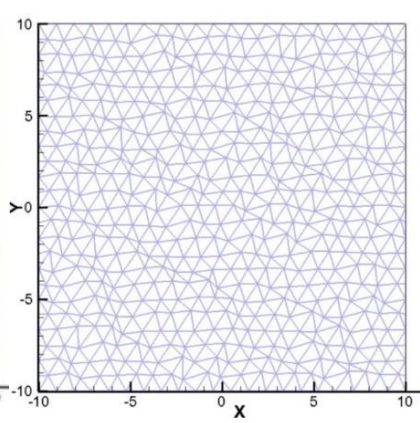

Distorted Grid (Type IV)

\section{Figure 3 Regular and Irregular Grid Types}

The grids of Type I are composed of equilateral triangular elements. Orthogonal girds of Type II refer to regular triangular grids derived from regular quadrilateral ones, in which square cells are decomposed in four triangular cells by diagonal splitting. Orthogonal grids of Type III are derived, in a similar fashion; two triangular cells are produced from each square one. As far as the Distorted grids of Type IV are concerned, grid irregularities are introduced by perturbing the grid nodes of an initial Type-I Equilateral Triangular grid from their original positions. The nodes' distortions are imposed with random shifts in each dimension, whereas the perturbation is defined as $0.4 r \Delta x ; r \in$ $[-0.5,0.5]$ is a random number and $\Delta x$ is the local mesh (cell) size along the given dimension. Representative grid types are depicted in Fig. 3.

A major requirement, in order to perform convergence studies with a sequence of refined grids, is the Consistency Refinement Property (Thomas et al., 2008). According to it, the maximum distance across the grid cells decrease consistently with increasing the total number of grid data points. For a given computational domain with dimensions $L_{x} \times L_{y}$ in the $x-$ and $y$-direction, respectively, a subdivision of $L_{x}$ by $N_{x}$ line segments is defined: $\Delta x=L_{x} /$ $N_{x}$. Depending on the grid type, the subdivision $\Delta y=L_{y} / N_{y}$ can be accordingly determined. A characteristic length is defined for each grid type as $\mathrm{h}_{N}=\sqrt{L_{x} \times L_{y}} / N, N$ being the number of data points. The Consistent Grid Refinement is performed when a reduction of $\Delta x / 2$ results in $h_{N}^{\prime} \simeq h_{N} / 2$ and $N^{\prime} \simeq 4 N$. Then, a series of increasingly fine grids from $20 \times 20,40 \times 40,80 \times 80,160 \times 160$ to $320 \times 320$ is employed.

\section{Numerical Results}

In order to measure the solution error, the volume weighted norm $L_{K}$ of the error was used, defined as (Delis et al., 2011; Delis and Nikolos, 2013)

$$
\left\|\mathrm{U}_{\mathrm{i}}-\mathrm{U}_{\mathrm{i}}^{\mathrm{ex}}\right\|_{L_{k(\Omega)}}=\left(\frac{\sum_{i=1}^{N}\left|\Omega_{i}\right|\left(U_{i}-U_{i}^{e x}\right)^{K}}{\sum_{i=1}^{N}\left|\Omega_{i}\right|}\right)^{\frac{1}{K}}
$$

where $U_{i}^{e x}$ is the exact solution and $U_{i}$ the numerical one, defined at node $i$ for the conserved variables $\left(\rho, \rho u, \rho v, \rho e_{T}\right)$, while $\Omega_{i}$ is the corresponding volume and $N$ is the number of data points. The errors were measured in two different norms $(K=2, K=\infty)$ between the numerical variables and their analytical counterparts at $t=60$.

Specifically, Fig. 4 presents the convergence results for $L_{2}$ norm for the conservative variables $\rho, \rho u, \rho e_{T}$ on each grid used, at $t=60$. Fig. 5 presents the corresponding convergence results for $L_{\infty}$ norm, at the same time instant. The results are compared with those of the $2^{\text {nd }}$ order MUSCL scheme, for the same grids. From the results it is evident that the discretization scheme can achieve the desired 3rd-order only for the Regular grid types while for the Irregular ones the order drops considerably.

Fig. 6 contains contour plots for $\rho$ of the analytic (dashed red line) and the numerical solution (solid blue line) at time instant $t=60$ (grid refinement 80 (top) and 320 
(bottom)) for the Equilateral grid (Type I). Similarly, Fig. 7 contains the corresponding contour plots for $\rho$ of the analytic and numerical solutions at the same time instant (grid refinement 80 (top) and 320 (bottom)) for the Distorted grid (Type IV). These plots confirm the convergence results and demonstrate clearly the effect of grid irregularity on the reduction of the discretization scheme's accuracy.

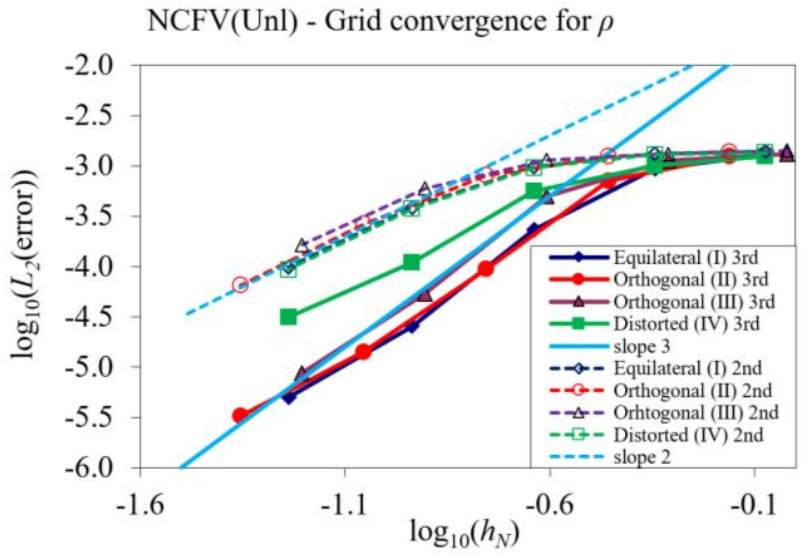

$\mathrm{NCFV}(\mathrm{Unl})$ - Grid convergence for $\rho u$

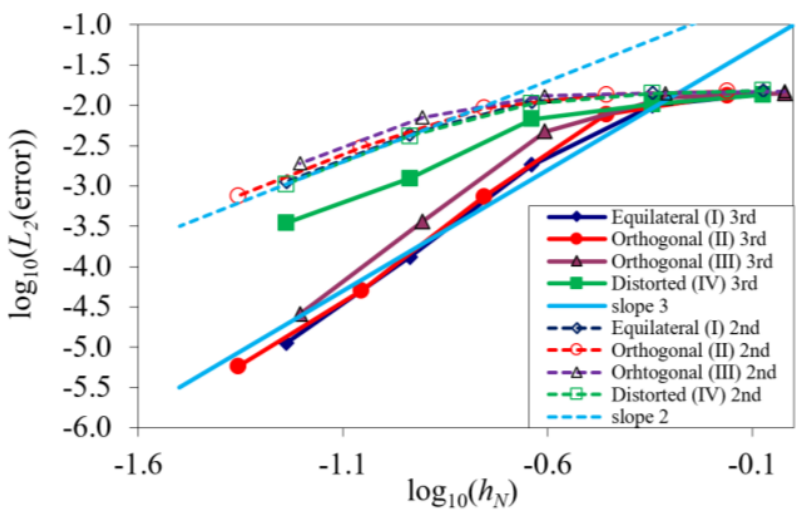

NCFV(Unl) - Grid convergence for $\rho e$

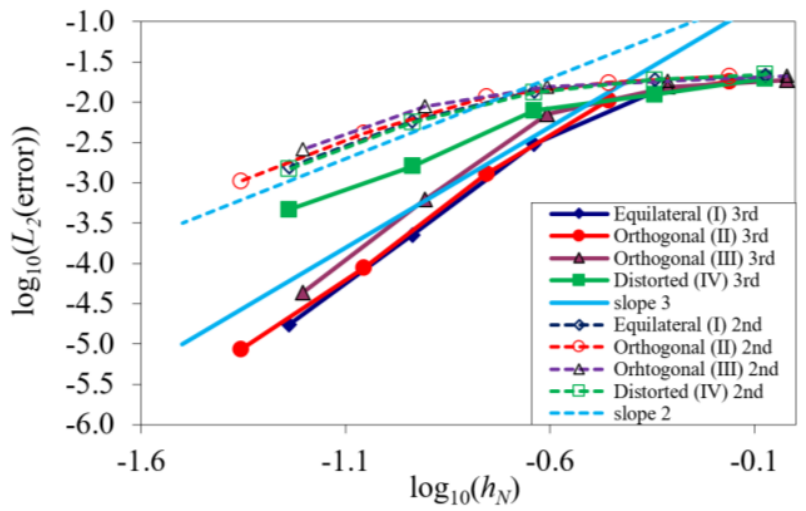

Figure 4 Convergence Results of $L_{2}$ Norm at $t=60$

The observed reduction in scheme's order for the irregular grids is due to two reasons. The first is the fact that the $U$-MUSCL scheme was developed for one-dimensional cases, and can be generalized in a straightforward manner only for structured regular grids in higher dimensions. However, for unstructured irregular grids its effectiveness considerably drops.

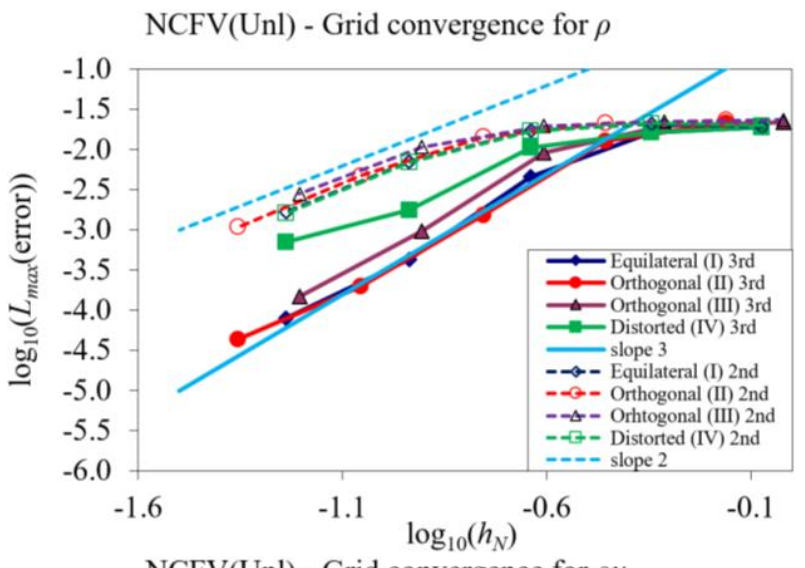

NCFV(Unl) - Grid convergence for $\rho u$

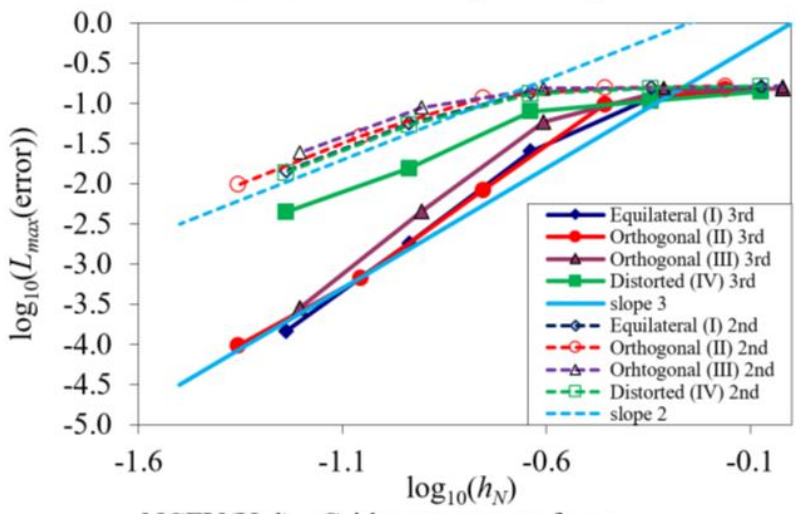

NCFV(Unl) - Grid convergence for $\rho e$

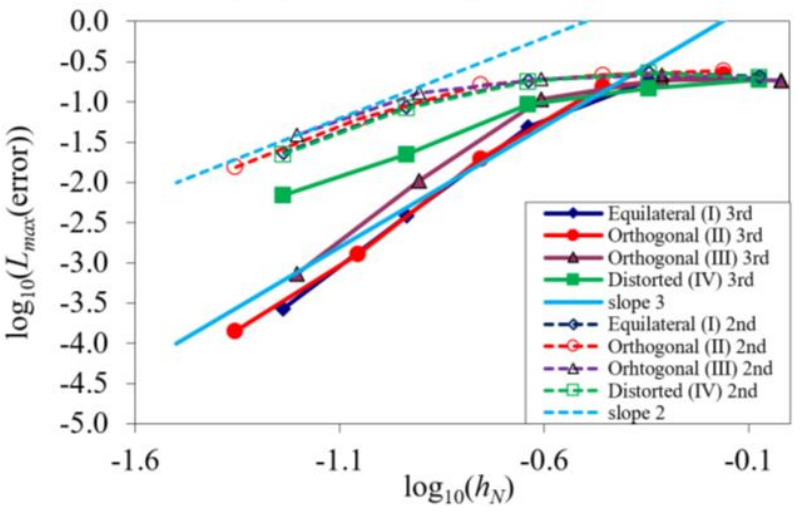

Figure 5 Convergence Results of $L_{\infty}$ Norm at $t=60$

The second reason, for the observed reduction in accuracy for the Irregular unstructured grids, is the inaccuracies in the gradients' computation, using the GreenGauss theorem, for highly distorted grids. The combination of the above reasons results in the observed undesirable effect. It is worth mentioning that all three Regular grids demonstrate a very similar behaviour, with respect to convergence results, despite their triangular elements being different, while they have different orientation, with respect to the vortex movement and its induced flow field.

Concerning the additional computational time required by the $3^{\text {rd }}$ order scheme, indicative results are provided for the $160 \times 160$ Equilateral grid at time $t=7$. For the 2 nd order MUSCL scheme a $C F L=0.8$ was used, while for the 3rd order $U$-MUSCL scheme a $C F L=1.5$ was used (due to 
the higher capacity of the $((\operatorname{SSP}-R K(5,4)))$. The additional time required by the 3 rd order scheme is about $11 \%$.
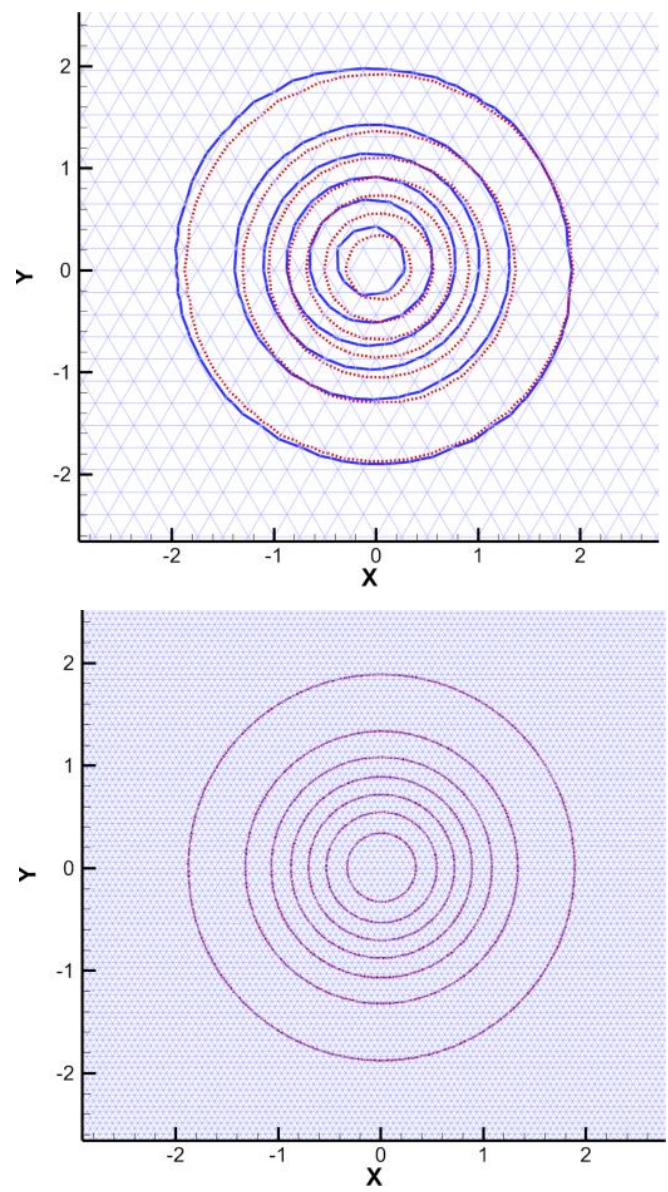

Figure 6 Contour Plots for $\rho$ of the Analytic (Red Dashed) and Numerical Solutions at $t=60$ (Grid Refinement 80 (top) and 320 (bottom), Type I grid)

\section{CONCLUSIONS}

In this study a recently-proposed high-order spatial discretization scheme was integrated into an existing academic solver for the numerical solution of the 2D compressible Euler equations. The discretized governing equations were solved with a Finite Volume Node-Centered scheme on unstructured triangular grids, whereas an upwind method was implemented for the computation of the inviscid fluxes, employing the Roe's approximate Riemann solver. A successive differentiation technique was utilized so as to compute the required high-order derivatives, using the Green-Gauss theorem. The formulation was based on the $U$ MUSCL reconstruction, which closely resembles the traditional MUSCL one. Additionally, a Strong Stability Preserving fourth-order five-stage Runge-Kutta method was used for time integration. The goal of this study was to test whether the promised 3rd-order of the scheme can be achieved for different types of unstructured grids, consisting of triangular elements; especially for irregular grid types.

The benchmark problem of an isentropic travelling vortex, with a well-known analytic solution, was encountered for the evaluation of the numerical accuracy. Using a controlled environment, with different unstructured grid types, through a successive grid refinement procedure, numerical simulations have been carried out for long time periods.
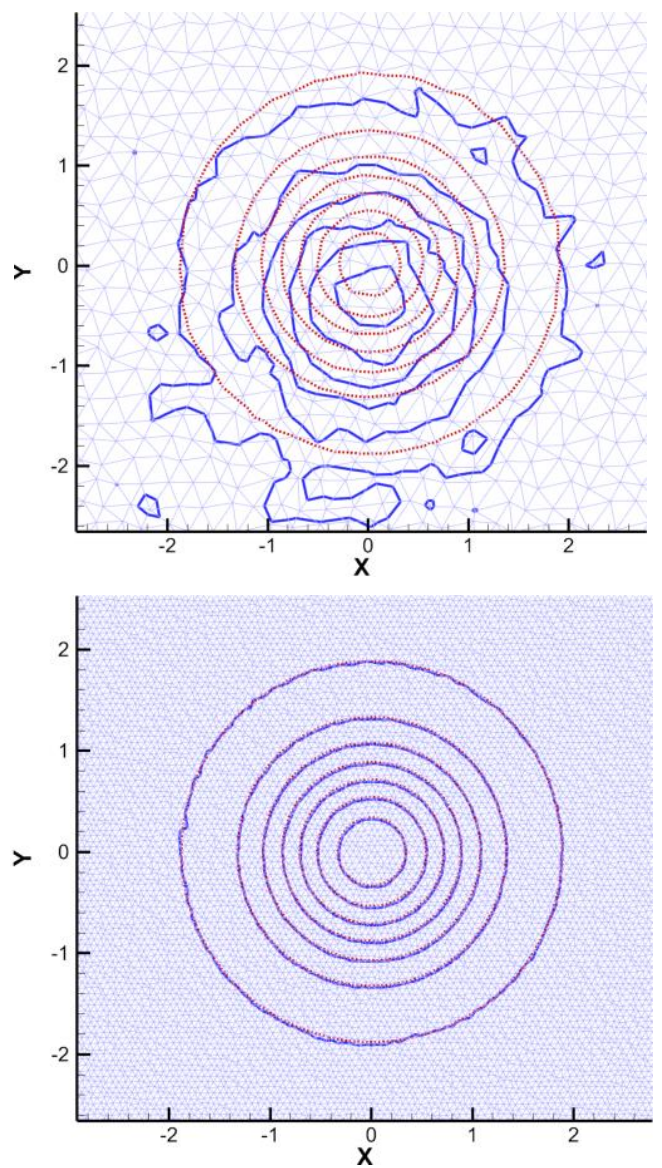

Figure 7 Contour Plots for $\rho$ of the Analytic (Red Dashed) and Numerical Solutions at $t=60$ (Grid Refinement 80 (top) and 320 (bottom), Type IV grid)

The following major conclusions can be drawn from the present study:

The employed scheme, in general, enhances the numerical accuracy of the existing solver. In terms of the convergence behaviour, the numerical results for regular grids obtain a satisfactory agreement with the promised 3rdorder accuracy of the discretization scheme. As expected, the distorted grids demonstrated an order reduction on the convergence rates, compared to the regular ones.

The experience of integrating the current numerical scheme into an existing academic solver showed an easy implementation without excessive efforts. Through the successive differentiation process, the computation of the high-order terms is feasible by exploiting the existing structure of the code with only slight modifications. The same routine that computes the field of first derivatives is utilized to obtain the field of the higher derivatives. This advantage seems quite preferable for parallel unstructured flow solvers (with domain decomposition), where the employment of the common high-order schemes is rather challenging. The proposed methodology requires minor 
modifications to the parallel implementation of the code; principally, the exchange of gradient information between the adjacent domains is required.

Concerning the extension of the presented methodology to $3 \mathrm{D}$, this is straightforward and easy, because it is compatible with the edge-based formulation. The successive differentiation process is easily applicable to $3 \mathrm{D}$, as it utilizes the existing subroutines for the computation of the $1 \mathrm{st}$ derivatives.

The memory requirements of the incorporated highorder module and the computation time of the numerical solution are kept low. The computation of the high-order terms requires only the allocation of 3 additional arrays in the case of a 3rd-order scheme, whereas the computational effort for the flux calculation in each iteration has a relatively low overhead, associated with the calculation of the above terms.

Finally, the implementation of the high-order module was conducted without taking into account quadrature rules in the computation of the numerical fluxes. Hence, it was restricted to the existing midpoint rule for the calculation of the flux integrals, albeit providing a significant improvement to the numerical solutions for regular grids. However, further work is required in order to solve the order-reduction problem for irregular unstructured grids.

Overall, the authors believe that the obtained results from the initial testing of the proposed methodology are very promising. The required modifications to an existing code are not extensive, while they are justified by the achieved order increase.

\section{REFERENCES}

Barth, T. (1992). Aspects of Unstructured Grids and FiniteVolume Solvers for the Euler and Navier-Stokes Equations. In: Proceedings of the AGARD-FDP-VKI special course at VKI. Rhode-Saint-Genese, AGARD, pp. 6.1-6.61.

Barth, T. (1993). Recent Developments in High Order KExact Reconstruction on Unstructured Meshes. In: 31st AIAA Aerospace Sciences Meeting. Reno, p. 16, doi: 10.2514/6.1993-668.

Blazek, J. (2001). Computational Fluid Dynamics: Principles and Applications. 1st ed. Kidlington: Elsevier Science.

Burg, C. (2005). Higher Order Variable Extrapolation for Unstructured Finite Volume RANS Flow Solvers. In: 17th AIAA Computational Fluid Dynamics Conference. Toronto, Canada, AIAA 2005-4999, doi: 10.2514/6.2005-4999.

Delis, A., Nikolos, I. and Kazolea, M. (2011). Performance and Comparison of Cell-Centered and Node-Centered Unstructured Finite Volume Discretizations for Shallow Water Free Surface Flows. Archives of Computational Methods in Engineering, 18(1), pp. 57-118, doi: 10.1007/s11831-011-9057-6.

Delis, A. and Nikolos, I. (2013). A Novel Multidimensional Solution Reconstruction and Edge-Based Limiting Procedure for Unstructured Cell-Centered Finite Volumes with Application to Shallow Water Dynamics. International Journal for Numerical Methods in Fluids, 71(5), pp. 584633, doi:10.1002/fld.3674.
Diskin, B. and Thomas, J. (2007), Accuracy Analysis for Mixed-Element Finite-Volume Discretization Schemes, National Institute of Aerospace, NIA Rept. No. 2007-08.

Gottlieb, S. (2005). On High Order Strong Stability Preserving Runge-Kutta and Multi Step Time Discretizations. Journal of Scientific Computing, 25(1), pp. 105-128, doi: 10.1007/s10915-004-4635-5.

Kallinderis, Y. and Ahn, H. (2005). Incompressible NavierStokes Method with General Hybrid Meshes. Journal of Computational Physics, 210(1), pp. 75-108, doi: 10.1016/j.jcp.2005.04.002.

Lygidakis, G. and Nikolos, I. (2015). Numerical Analysis of Flow Over the NASA Common Research Model Using the Academic Computational Fluid Dynamics Code Galatea. Journal of Fluids Engineering, 137(7), 071103, doi: 10.1115/1.4029730.

Persson, P. (2012). High-Order Navier-Stokes Simulations Using a Sparse Line-Based Discontinuous Galerkin Method. In: 50th AIAA Aerospace Sciences Meeting including the New Horizons Forum and Aerospace Exposition. Nashville, p. 12, doi: 10.2514/6.2012-456.

Roe, P. (1981). Approximate Riemann Solvers, Parameter Vectors and Difference Schemes. Journal of Computational Physics, 43(2), pp. 357-372, doi: 10.1016/00219991(81)90128-5.

Ruuth, S. (2006). Global Optimization of Explicit StrongStability-Preserving Runge-Kutta Methods. Mathematics of Computation, 75(253), pp. 183-207, doi: 10.1090/S00255718-05-01772-2.

Spalart, P. and Venkatakrishnan, V. (2016). On the Role and Challenges of CFD in the Aerospace Industry. The Aeronautical Journal, 120(1223), pp. 209-232, doi: 10.1017/aer.2015.10.

Sweby, P. (1984). High Resolution Schemes Using Flux Limiters for Hyperbolic Conservation Laws. SIAM Journal on Numerical Analysis, 21(5), pp. 995-1011, doi: 10.1137/0721062.

Thomas, J., Diskin B. and Rumsey, C. (2008). Towards Verification of Unstructured-Grid Solvers. In: 46th AIAA Aerospace Sciences Meeting and Exhibit. Reno, p. 13, doi: 10.2514/6.2008-666.

Van Albada, G., Van Leer, B. and Roberts, W. (1982). A Comparative Study of Computational Methods in Cosmic Gas Dynamics. Astronomy and Astrophysics, 108(1), pp. 4684.

Van Leer, B. (1979). Towards the Ultimate Conservative Difference Scheme, V. A Second Order Sequel to Godunov's Method. Journal of Computational Physics, 32(1), pp. 101136, doi:10.1016/0021-9991(79)90145-1.

Yang, H., Chen, Z., Przekwas, A. and Dudley, J. (2014). A High-Order CFD Method Using Successive Differentiation. Journal of Computational Physics, 281, pp. 690-707, doi: 10.1016/j.jcp.2014.10.046.

Yang, H. and Harris, R. (2016). Development of VertexCentered High-Order Schemes and Implementation in FUN3D. AIAA Journal, 54(12), pp. 3742-3760, doi: 10.2514/1.J054561. 